\title{
Demonstration of a Longitudinal Concentration Gradient Along Scala Tympani by Sequential Sampling of Perilymph from the Cochlear Apex
}

\author{
Robert Mynatt, ${ }^{1}$ Shane A. Hale, ${ }^{2}$ Ruth M. Gill, ${ }^{2}$ Stefan K. Plontke, ${ }^{3}$ and Alec N. Salt $^{2}$ \\ ${ }^{1}$ Department of Otolaryngology, St. Louis University School of Medicine, St. Louis, MO 63110, USA \\ ${ }^{2}$ Department of Otolaryngology, Washington University School of Medicine, 660 South Euclid Avenue, Box 8115, St. Louis, \\ MO 63110, USA \\ ${ }^{3}$ Department of Otorhinolaryngology, Head and Neck Surgery and Tübingen Hearing Research Center (THRC), University of \\ Tübingen, D-72076, Tübingen, Germany
}

Received: 15 December 2005; Accepted: 17 March 2006; Online publication: 22 April 2006

\begin{abstract}
Local applications of drugs to the inner ear are increasingly being used to treat patients' inner ear disorders. Knowledge of the pharmacokinetics of drugs in the inner ear fluids is essential for a scientific basis for such treatments. When auditory function is of primary interest, the drug's kinetics in scala tympani (ST) must be established. Measurement of drug levels in ST is technically difficult because of the known contamination of perilymph samples taken from the basal cochlear turn with cerebrospinal fluid (CSF). Recently, we reported a technique in which perilymph was sampled from the cochlear apex to minimize the influence of CSF contamination (J. Neurosci. Methods, doi: $10.1016 / \mathrm{j}$.jneumeth.2005.10.008). This technique has now been extended by taking smaller fluid samples sequentially from the cochlear apex, which can be used to quantify drug gradients along ST. The sampling and analysis methods were evaluated using an ionic marker, trimethylphenylammonium (TMPA), that was applied to the round window membrane. After loading perilymph with TMPA, 10 $1-\mu \mathrm{L}$ samples were taken from the cochlear apex. The TMPA content of the samples was consistent with the first sample containing perilymph from apical regions and the fourth or fifth sample containing
\end{abstract}

Correspondence to: Alec N. Salt · Department of Otolaryngology · Washington University School of Medicine $\cdot 660$ South Euclid Avenue Box 8115 St. Louis, MO 63110, USA. Telephone: +1-3143627560; fax: +1-314-3627522; email: salta@msnotes.wustl.edu perilymph from the basal turn. TMPA concentration decreased in subsequent samples, as they increasingly contained CSF that had passed through ST. Sample concentration curves were interpreted quantitatively by simulation of the experiment with a finite element model and by an automated curve-fitting method by which the apical-basal gradient was estimated. The study demonstrates that sequential apical sampling provides drug gradient data for ST perilymph while avoiding the major distortions of sample composition associated with basal turn sampling. The method can be used for any substance for which a sensitive assay is available and is therefore of high relevance for the development of preclinical and clinical strategies for local drug delivery to the inner ear.

Keywords: perilymph sampling, perilymph kinetics, pharmacokinetics, round window membrane, inner ear drug delivery, computer simulation Abbreviations: ST - scala tympani; CSF - cerebrospinal fluid; RW-round window; RWM-round window membrane; TMPA - trimethylphenylammonium

\section{INTRODUCTION}

To establish the pharmacokinetics of a drug in the inner ear fluid spaces, it is necessary to collect and analyze fluid samples from the inner ear. The validity of such measures depends on where the sample is taken from and how it is obtained (recently reviewed by Salt and Plontke 2005). A serious technical difficulty arises because of the anatomic connection 
between cochlear perilymph and the cerebrospinal fluid (CSF) through the cochlear aqueduct. The aqueduct opens into the basal turn of scala tympani (ST) near the round window membrane (RWM). When the cochlea is in the normal, sealed state, volume flow through the aqueduct is very low and makes little contribution to perilymph homeostasis (Ohyama et al. 1988). In contrast, when the bony otic capsule is perforated or as a fluid sample is withdrawn, the perilymph is rapidly replaced by a flow of CSF entering the ST through the aqueduct (Moscovitch et al. 1973; Salt and Stopp 1979; Ohyama et al. 1988). Fluid samples taken from the basal turn of ST are at high risk for being contaminated with CSF (Hara et al. 1989; Scheibe et al. 1984). Based on marker measurements in perilymph before and during sampling through the RWM of guinea pigs, it was calculated that $2-\mu \mathrm{L}$ samples were composed of $50 \%$ perilymph and 50\% CSF (Salt et al. 2003). Larger, $10-\mu \mathrm{L}$ samples, which have been collected in a number of recent pharmacokinetic studies, were estimated to contain only $15 \%$ perilymph. A high degree of perilymph dilution with CSF creates difficulties in interpreting sample concentrations, as the sample depends on both perilymph and CSF compositions. Larger perilymph volumes can be obtained with less CSF contamination when samples were taken at a greater distance from the cochlear aqueduct. Samples taken from the vestibule (Hoffer et al. 1997) or from scala vestibuli appear to be less influenced by CSF contamination. However, for those studies that are focused on the auditory effects of applied drugs, the kinetics of drug in ST is of primary interest, as drugs present in ST have access to the auditory hair cells and to the lateral wall tissues of the cochlea. The known difficulties associated with sampling from ST cannot therefore be bypassed by sampling near the vestibule.

One solution to the problem of sampling from ST was recently provided by a novel technique of sampling from the cochlear apex. The method utilized a silicone cup that was constructed around the apex. When the bony capsule was perforated within the cup, all the fluid emerging could be collected without any loss to the middle ear spaces (Salt et al. 2005). The fluid collected from the apex represents fluid that is being displaced by CSF entry through the cochlear aqueduct. Prior studies in which perilymph was sampled from the basal turn of ST and which would have been very sensitive to flow originating from the vestibule or scala vestibuli failed to detect any contribution other than from CSF (Salt et al. 2003). Similarly, longitudinal flow measurements in ST after perforation of the RWM detected no basally directed flow toward the perforation site (Salt et al. 1991). It was concluded that the cochlear and vestibular systems did not produce fluid in volume when they were perforated. Thus, the dominant mechanism by which apical samples originate is through perilymph displacement by CSF entry through the aqueduct. In the previous analysis of $10-\mu \mathrm{L}$ samples collected from the cochlear apex, findings were consistent with the samples consisting of virtually all the ST contents (estimated volume 4.7 $\mu \mathrm{L}$ ) with the remaining volume made up of CSF that had "rinsed through" the ST (Salt et al. 2005). In that study, the samples from the apex contained virtually all of the solute that had been present in ST prior to sampling. The interpretation of the composition of large samples $(10 \mu \mathrm{L})$ taken from the apex is therefore subject to smaller errors than are samples of similar volume taken from the basal turn of ST.

In the current study, a further development of the apical sampling method is presented, which greatly adds to the scientific value of the measurements by providing detailed information about the distribution of drugs along ST. Instead of taking one large sample from the apex, we have sequentially obtained 10 samples, each of approximately $1-\mu \mathrm{L}$ volume, which were analyzed independently. This enhancement requires a very sensitive assay method capable of dealing with samples of limited volume.

\section{METHODS}

\section{Animal preparation}

Sequential sampling of fluid from the cochlear apex was performed in eight pigmented, NIH strain guinea pigs. Animals were anesthetized with 100 $\mathrm{mg} / \mathrm{kg}$ sodium thiobutabarbital (Inactin, Sigma, St. Louis, MO, USA) and given supplementary doses regularly as required via an intravenous line in the external jugular vein. Animals were given pancuronium bromide as a muscle relaxant and artificially respired through a tracheal cannula with end-tidal $\mathrm{CO}_{2}$ level maintained near $38 \mathrm{~mm} \mathrm{Hg}(5 \%)$. Heart rate and vascular $p \mathrm{O}_{2}$ were monitored continuously by Surgivet pulse oximeter (Waukesha, WI, USA). Rectal temperature was maintained at $38^{\circ} \mathrm{C}$ with a thermistor-controlled DC-powered heating blanket. The animal was mounted in a head holder, and the cochlea was exposed by the ventrolateral approach.

\section{Preparation of the cochlear apex for fluid sampling}

Complete illustrated details of the methods used to perform apical fluid sampling are presented elsewhere (Salt et al. 2005) and are only given briefly here. The mucosae on the cochlea were cleared from the two apical turns using a small cotton swab, and the 
bone at the apex was allowed to dry. A droplet of thin cyanoacrylate adhesive was applied to the bone and was spread as thinly as possible over the apex using a plastic fiber. Two-part silicone adhesive (WPI KwikCast) was then applied to the apical region, keeping the adhesive as thin as possible over the site to be perforated. Around the periphery, silicone was built up in multiple layers until a "cup" shape was formed.

\section{TMPA loading of perilymph}

Trimethylphenylammonium (TMPA) was applied to perilymph by irrigating solution containing $20 \mathrm{mM}$ TMPA across the RWM at $5 \mu \mathrm{L} / \mathrm{min}$. The background medium for this solution was an artificial perilymph with reduced sodium containing (in $\mathrm{mM}$ ): $\mathrm{NaCl}, 125 ; \mathrm{KCl}, 3.5 ; \mathrm{NaHCO}_{3}, 25 ; \mathrm{MgCl}_{2}, 1.2 ; \mathrm{CaCl}_{2}$, 1.3; $\mathrm{NaH}_{2} \mathrm{PO}_{4}, 0.75 ; \mathrm{C}_{6} \mathrm{H}_{12} \mathrm{O}_{6}, 5.0$, so that the addition of $20 \mathrm{mM}$ TMPACl resulted in a normal solution osmolarity (approximately $291 \mathrm{mOsm} / \mathrm{L}$ ). TMPA solution was applied to the RWM for $2 \mathrm{~h}(n=$ $7)$ or $2.5 \mathrm{~h}(n=1)$. In three animals, the RWM was made more permeable to TMPA by blowing desiccated air into the bulla for approximately $20 \mathrm{~s}$. A brief exposure to desiccated air increases the RWM permeability to TMPA (Salt et al. 2005). During TMPA delivery, wicks were placed in the bulla to ensure that the TMPA solution did not contaminate the sampling site.

\section{Sampling from the cochlear apex}

The principle underlying sequential sampling from the cochlear apex is illustrated in Fig. 1. The example shows the cochlea with a gradient of drug along ST with the highest concentration in the basal turn. When fluid samples are taken from the apex, the fluid lost from the cochlea is replaced by CSF, entering the ST in the basal turn through the cochlear aqueduct. The initial sample (1) contains fluid that originated in the most apical regions of ST. Each successive sample (2-4) contains perilymph from a more basal region, up to the point where CSF contributes significantly to the sample. An increasing concentration of substance in the first few samples taken from the apex is therefore qualitatively indicative of a basal to apical gradient of the substance along the ST. This figure is simplified and does not include the diffusion of substance into CSF from the high concentration fluid or from other cochlear compartments that have become loaded with the substance.

Prior to sampling, all drug solution was removed from the auditory bulla and the RW niche area using tissue wicks. Care was taken to avoid touching the RWM with the wicks during drying. The bulla and the silicone cup over the sampling region were also rinsed
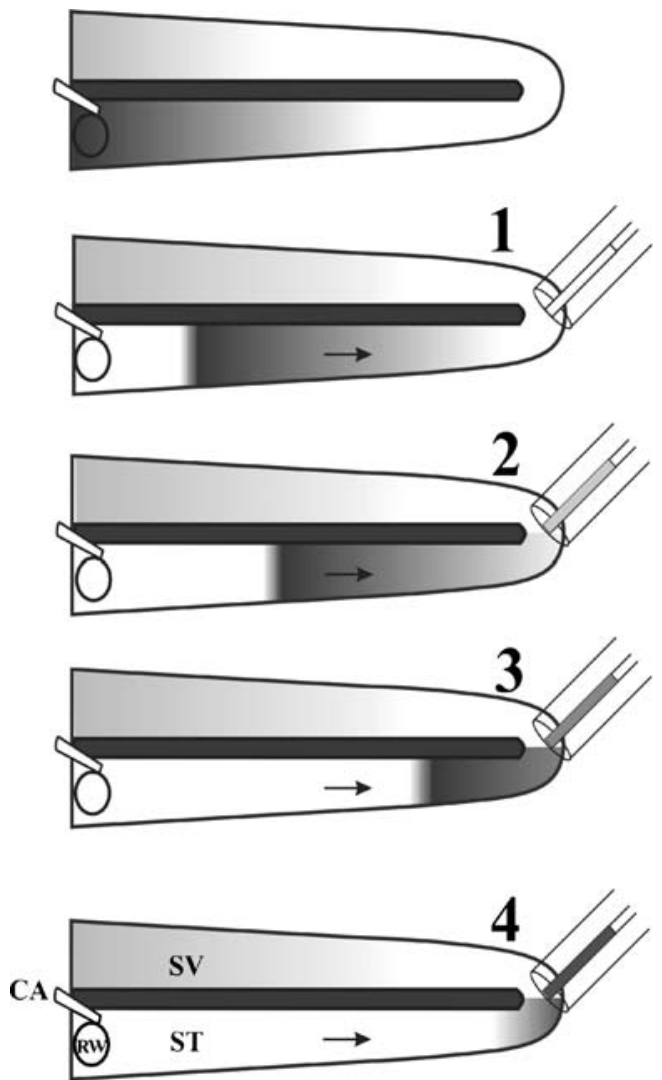

FIG. 1. Schematic of the unwound cochlea before and during fluid sampling from the cochlear apex. Prior to sampling (upper panel), a concentration gradient of drug exists along scala tympani (ST). When the apex is perforated, the fluid efflux collected in a capillary is initially from apical regions (1). The fluid volume lost from the cochlea is replaced by CSF entering the basal turn of ST through the cochlear aqueduct (CA). Subsequent samples (2-4) contain fluid that originated from progressively more basal regions of ST, up to the point where the sample contains a significant amount of CSF. Further samples (not shown) increasingly contain CSF that has "rinsed through" ST.

twice with lactated Ringer's just in case the site had been inadvertently contaminated. The procedures of drying the bulla and rinsing the sampling site took 58 min. A small perforation (50- to $100-\mu \mathrm{m}$ diameter) was then made in the bone over scala vestibuli at the apex using a $30^{\circ}$ pick (Storz N170580, $1 / 3 \mathrm{~mm} \mathrm{30^{ \circ }}$ oval window pick, Bausch \& Lomb Surgical, St. Louis, MO, USA). Upon perforation, fluid immediately started emerging, driven by CSF pressure, and formed a bead within the hydrophobic silicone cup. A calibrated 5- $\mu \mathrm{L}$ capillary micropipette (VWR \# 53432706, S. Plainfield, NJ), marked with permanent ink at the $1-\mu \mathrm{L}$ location, was held by hand in contact with the fluid at the apex, so that fluid was drawn into the capillary. The time taken to accumulate a nominally $1-\mu \mathrm{L}$ sample with the capillary touched to the apex averaged $33.1 \mathrm{~s}$ (SD $16.8 \mathrm{~s}, n=80$ ). The negative pressure in the capillary partially contributed to this 
flow rate, although the capillaries were blunt and did not seal tightly to the apex. When the fluid meniscus reached the $1-\mu \mathrm{L}$ mark, the capillary was removed and replaced with a new one. Changing capillaries took just a few seconds, so fluid volume accumulating during this time was a minor fraction of each sample. In addition, fluid emerged from the cochlea more slowly without the capillary in contact with the apex because of the convex meniscus (positive pressure) of the fluid droplet in contact with silicone relative to the concave meniscus (negative pressure) in the capillary. Obtaining the correct sample volume was based on visual observation of the meniscus in the capillary as the sample accumulated rather than the timing of the sample. Ten separate samples were taken sequentially from the apex in this manner. Documentation of the specific timing of sample collection in each experiment was required for subsequent simulation of the experiment and was performed by an oral commentary recorded with a time record on the computer (Audacity, http:// audacity.sourceforge.net/). Immediately after each sample was collected, its exact volume was determined by measuring the sample length relative to the commercially applied $5-\mu \mathrm{L}$ calibration mark with a calibrated dissecting microscope.

\section{Analysis of samples}

The TMPA content of each sample was measured in vitro at room temperature with a TMPA-selective microelectrode. The electrodes were made on the day of use using methods detailed elsewhere (Salt et al. 2005) and calibrated before and after use in seven standards containing $0,1,3.33,10,33.33,100$, and $1000 \mu \mathrm{M}$ TMPA in a background of artificial perilymph. The mean slope of the electrode response to TMPA was $62.5 \mathrm{mV} /$ decade (SD 2.8, $n=8$ ), and the "detection limit" (the limit above which a linear electrode response can be assumed) for TMPA was $1.11 \mu \mathrm{M}$ (SD 0.16, $n=8)$. Measurements were performed below the detection limit by fitting a nonlinear calibration curve (Salt and Vora 1991). Samples taken from the animal were added to $25 \mu \mathrm{L}$ of artificial perilymph as a dilutent. The 10 diluted samples together with similar volumes of 0,1 , and 10 $\mu \mathrm{M}$ TMPA standards were each put in separate wells in a Teflon block. A TMPA-selective electrode was used to make the following measurements in sequence: (1) large-volume standards; (2) each of the small-volume standards in the block; (3) each of the 10 samples; (4) each of the standards in the block again; (5) each of the 10 samples again; and (6) the standards in the block for the third time. Iondependent potential and time data were logged by computer. Based on the repeated measurements, small changes in the samples and standards because of evaporation could be compensated. The measured TMPA concentrations were then corrected for the dilution based on the individual sample volume and volume of dilutent. All TMPA concentrations presented represent that in the undiluted sample from the cochlea.

\section{Analysis by simulation of each experiment}

The data from each experiment were interpreted by simulation of the specific experimental conditions, including the timing and actual volumes of the 10 apical samples taken. This was performed with a modified version of the finite-element cochlear fluids simulation program, made available at http://oto. wustl.edu/cochlea/. The program was modified to incorporate the volume flows and sample accumulation associated with sampling from the cochlear apex, taking into account the total volume and rate of accumulation of each individual sample. The diffusion coefficient used for TMPA was $1.01 \times 10^{-9} \mathrm{~m}^{2} / \mathrm{s}$. The loading of ST with TMPA was accomplished by simulating TMPA entry across the RW membrane. One output of the model was the concentration values for the 10 samples taken from the cochlear apex, which were compared with the measured concentration values. The sum of squares of differences between calculated and measured values were used as an indicator of how well the model fit the data. The model parameters systematically varied to optimize fit were: (1) RWM permeability; (2) TMPA clearance from ST; (3) the rate of longitudinal perilymph flow along the ST throughout the loading period, i.e., prior to apical perforation; and (4) the cross-sectional area and accessibility of "parallel compartments" to ST. The half time of clearance from scala vestibuli was set to twice that of ST. The "parallel compartment" represents the fluid and tissue spaces in the cochlea that are not included in the documented scala areas, specifically the spiral ligament, organ of Corti, and spiral ganglion. The incorporation of TMPA diffusion into such spaces during loading, and the diffusion out of these spaces as the perilymph is replaced by CSF during fluid sampling, are necessary to account for the measured rate of TMPA decline during sampling. This differs from clearance from ST, which represents the loss of TMPA to the vascular system, regarded as an infinite pool with zero TMPA concentration. TMPA lost from ST by clearance never reenters the perilymph.

For the parameter set that provided the best fit between the simulated and real samples, the model also provided the calculated TMPA concentration profile along the length of ST just prior to sampling. This profile was used to determine the TMPA gradient 
along ST. Gradients are expressed as the logarithm of the ratio between basal and apical concentration values; for example, with a basal concentration of 1000 and an apical concentration of 10 , the gradient would be $\log (1000 / 10)=\log (100)=2$.

\section{Analysis by curve fitting}

This analysis was performed using an automated Microsoft Excel spreadsheet (available for download at http://oto.wustl.edu/cochlea/apicalsamples/) that utilized the sample concentrations and volumes for each experiment. Analysis proceeded in the following sequence:

(1) Based on ST cross-sectional area versus distance data, the segment of ST volume that represented each specific sample was calculated. For this study, scala area data for the guinea pig were used, but the spreadsheet incorporates ST crosssectional area data for seven animal species, including humans. The distance calculated was that corresponding to the location of the midpoint of the sample on a volume basis. Each subsequent sample location was calculated taking into account the prior sample volumes. This analysis utilized the specific sample volumes for the experiment and allowed sample concentrations to be plotted as a function of distance along the cochlea. The algorithm selected and utilized only those samples that were calculated to originate from locations apical to the site of the cochlear aqueduct (1.1 $\mathrm{mm}$ in guinea pigs).

(2) Samples originating near the base of the cochlea were then corrected for interaction with CSF during sampling. This interaction not only includes dilution, but also the diffusion of TMPA from high concentration regions into lower concentration, CSF-diluted locations. Correction was based on simulations with uniform distribution of substance along the scala, using different sample volume paradigms. An exponential function that closely fitted these data was established in which the corrected sample concentration $\left(C_{\mathrm{s}}{ }^{*}\right)$ at distance (dist) along ST was:

$$
C_{s}^{*}=C_{s} \times a\left(e^{-b(\mathrm{dist}-d)}+1\right)
$$

The parameters, derived by best fit to simulated samples, were $a=1.26, b=3.01$, and $d=0.96$.

In practice, for $1-\mu \mathrm{L}$ samples from the guinea pig, this correction only influenced the fourth sample, as detailed below.

(3) A nonlinear function approximating the typical concentration profile along ST was fitted to the concentration versus distance data using the "solver" curve-fitting capability of Microsoft Ex- cel. This fitted function allowed concentration to be extrapolated from the sample points to the basal and apical limits of ST, permitting a gradient to be calculated that represented the entire scala length. The function is a curve in which the concentration $(C)$ closely fits the concentration profiles along the cochlea but is defined by only two parameters. This is important when the curve is being fitted to only a limited number of sample points (typically four in this study). The fitted function was

$$
C=10^{\left(\log \left(C_{\text {int }}\right)-s \times \operatorname{dist}^{(b)}\right)}
$$

where the parameters $C_{\text {int }}$ (the concentration intercept) and $s$ (a slope factor) were the two parameters varied to fit the data. The exponent (b) was established by fitting the function to concentration profiles established by finite-element simulation of sampling experiments and was found to average 1.59 . It was subsequently held constant at this value. The use of a function of concentration versus distance, fitted to the sample data, allowed the basal and apical concentrations, and the gradient to be estimated. The above algorithms were based on experimental data and simulations, as detailed further in the results below. They were incorporated into a spreadsheet macro and were performed in a fully automated manner. The intent of this analysis was to provide a straightforward method to interpret sequential apical sampling data that did not require finite-element modeling.

The experimental protocols for this study were approved by the Animal Studies Committee of Washington University (approval number 20040209).

\section{RESULTS}

The measured TMPA content of samples taken from the cochlear apex following a 2-h or longer loading period is summarized in Fig. 2. The data from normal and RW permeabilized animals are shown in the left and center panels, respectively. In all cases, the TMPA content of the initial sample was low, followed by a progressive increase in sample concentration reaching a maximum in the fourth or fifth sample. Following the peak, the TMPA content declined progressively with each successive sample. These data are qualitatively consistent with the existence of a basal-apical concentration gradient of TMPA at the time of sampling, comparable to that illustrated in Fig. 1. The right panel of Fig. 2 shows the mean curves for normal and RW permeabilized animals after normalizing the amplitude of each 

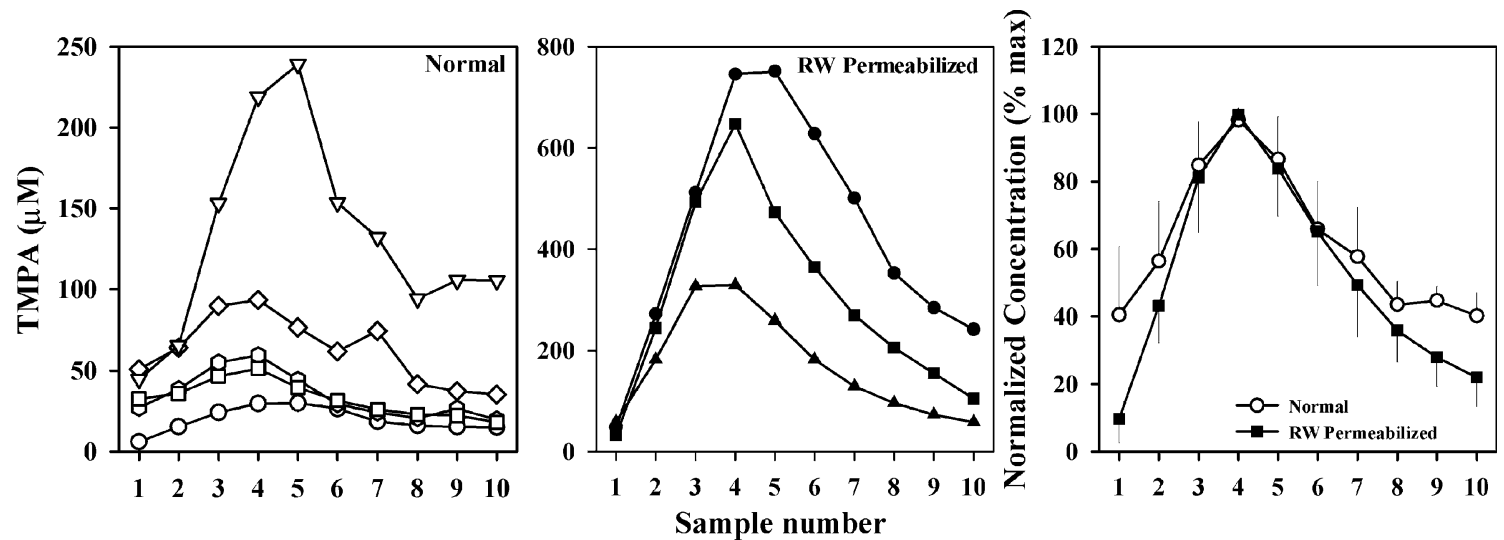

FIG. 2. Summary of the TMPA concentrations of 10 fluid samples taken sequentially from the cochlear apex. In the left and middle panels, each curve is a different experiment, shown with different symbols. In all curves, the initial sample had low concentration, reflecting its apical origins, increasing to a peak concentration at the fourth or fifth sample, representing fluid from more basal regions. Data are shown for experiments in which TMPA was applied without round window membrane (RWM) manipulation (left panel) and following RWM permeabilization (middle panel), which resulted in higher absolute perilymph concentrations of TMPA. Note that the left and middle panels have different concentration scales. The right panel shows the mean curves (bars indicate SD) for both groups after amplitude normalization.

curve by setting the maximum to $100 \%$. The two normalized curves showed a similar peak location and shape, except for the lower concentration samples before the peak (samples 1 and 2) and after the peak (samples 9 and 10). This difference was caused by the difficulty in resolving lower concentration values when the total amount of TMPA in the cochlea was low. It was also the rationale for including RW permeabilized animals in the study to produce TMPA levels high enough so that the lower concentrations in the early and late samples could be more accurately resolved.

Our finite element model of the cochlear fluids provided a tool for the quantitative interpretation of sequential sample data. Figure 3 shows four examples of different longitudinal drug gradients calculated along ST for different application conditions and clearance rates. The application conditions were intended to produce a range of drug gradients along the modeled ST, from steep (upper panel) to absent (lower panel). In each case, the right panel shows the calculated concentrations of 10 sequentially obtained $1-\mu \mathrm{L}$ samples, simulated as taken from the cochlear apex. The first four points approximately represented the longitudinal concentration gradient along ST in reverse order, with the first sample representing the apical concentration and the fourth sample representing the basal concentration. With a steep gradient along the scala (upper right panel), the difference between the first and fourth samples was greater than when no gradient existed (lower right panel) with a progression between the four application conditions. Thus, the curves based on sequentially obtained samples provide a representation of the drug gradient along ST. The simulation for the no-gradient situation (lower panel) also showed that the fourth sample was at a lower concentration than the known perilymph concentration. Even considering that the volume of ST is $4.7 \mu \mathrm{L}$, the calculated fourth 1- $\mu \mathrm{L}$ sample showed influence of drug dilution with CSF. In the model, CSF enters the basal turn of ST $1.1 \mathrm{~mm}$ from the base (Ghiz et al. 2001), but its influence spreads as the perilymph and CSF mix by diffusion. One consequence of perilymph and CSF intermixing by diffusion during sampling is that the gradient of drug indicated by the samples substantially underestimated the actual gradient along the scala. This was less of a problem when gradients were low (lower two rows in Fig. 3) but became increasingly significant when steeper gradients were present along the scala. For the steep gradient (upper row), the gradient determined from the ST profile (left panel) was $\log (610 / 0.98)=2.8$. In contrast, the gradient calculated by taking the highest sample (sample $4=253$ ) and the first apical sample (19) was $\log (253 / 19)=1.1$. Thus, longitudinal gradients of drugs could be seriously underestimated without a more detailed analysis of the sample data.

Each experiment was analyzed individually using the finite-element fluids model to simulate cochlear loading and sampling, in which the simulated samples were best fit to the experimental data. Figure 4 shows an example experiment in which the simulated samples are compared with the experimental samples (Fig. 4A). The concentration profile along ST that existed just prior to sampling in the simulation is shown by the solid line in Fig. 4B. Based on this curve, the longitudinal gradient of TMPA along ST in this experiment was calculated to be 5.97 (log units). A basal turn concentration of $1739 \mu \mathrm{M}$ was required to obtain a peak concentration in sample 4 near the 


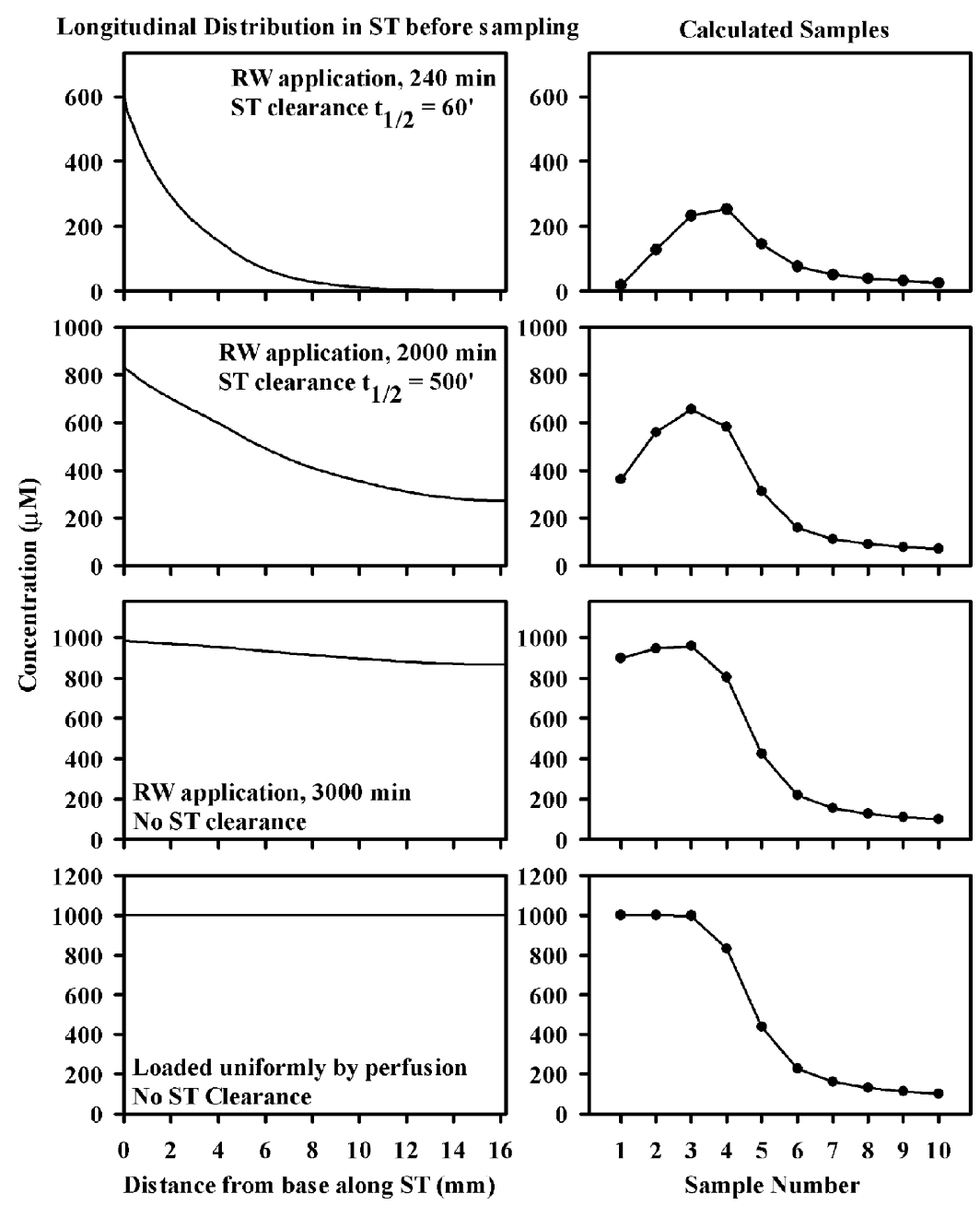

FIG. 3. Simulation of sampling from the cochlear apex. In each row, the longitudinal gradient of substance along ST (left panel) is compared with the 10 simulated samples obtained for that profile (right panel). Different application conditions were used to produce four different gradients from steep gradient (upper row) to none (lower row).

experimental value of $647 \mu \mathrm{M}$. This analysis confirmed that substantial errors could occur if the drug gradient was inferred without detailed analysis of the samples. The necessity for simulating the experiments was further demonstrated by calculating the TMPA concentrations in the ST segments representing the volumes of the first four samples, shown as triangles in Fig. 4B and $\mathrm{C}$ and shown pictorially in Fig. 4D. The simulation demonstrated that the samples differed substantially from the scala segments from which they originated, as shown in Fig. 4C. The mechanisms contributing to the observed differences include the dilution of basal samples by CSF and the loss of TMPA into other compartments of the cochlea as the solution moves apically along the cochlear spiral during sampling (Salt et al. 2005).

Concentration gradients calculated by simulation of the eight experiments in this study are summarized in Fig. 5. The original profiles along ST are shown on a linear scale (left panel) and normalized on a logarith- mic scale (right panel). The basal-apical gradient derived from this analysis is indicated for each curve on the logarithmic plot. The mean gradient was 4.27 (SD 1.77, $n=8$ ). The mean simulation parameters (with SD in parentheses) for the eight experiments were as follows: ST clearance half time $=74 \mathrm{~min}(31)$; volume flow along $\mathrm{ST}=19 \mathrm{~nL} / \mathrm{min}$ (15), apically directed; compartment parallel to ST area $=0.27 \mathrm{~mm}^{2}$ (0.07); accessibility $0.22(0.07)$ relative to free diffusion. The round window permeability averaged 0.93 $\times 10^{-8} \mathrm{~m} / \mathrm{s}(0.75, n=5)$ in normal animals and $12.1 \times$ $10^{-8} \mathrm{~m} / \mathrm{s}(7.04, n=3)$ in RW permeabilized animals. The volume flow rate along ST represented the extremely slow flow occurring during the entire TMPA loading period when the cochlear apex was intact. The flow rates during sampling, which were defined by the sample volumes and the rate to accumulate each sample, were approximately 100 times this rate.

The use of the finite element model to interpret sample data was time consuming, requiring from 20 

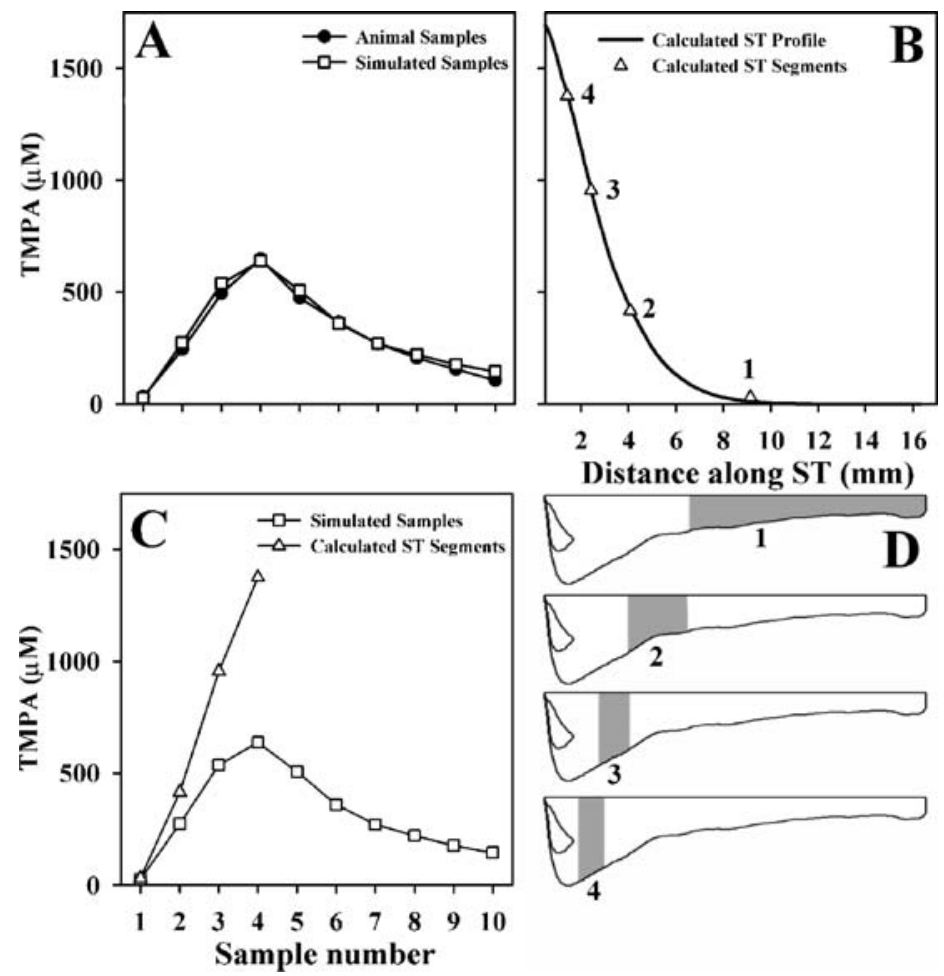

FIG. 4. Interpretation of apical sample concentrations in one experiment (ASP12) using the finite element simulator. (A) Experimental sample data compared with concentrations derived by simulation. (B) The scala tympani profile that existed just before sampling in the simulation. The basal and apical concentrations of this profile were 1739 and $0.0019 \mu \mathrm{M}$, respectively, indicating a basal-apical gradient of $9.4 \times 10^{5}$, which is $5.97 \log$ units. (C) Concentrations of the "volume segments" corresponding to the first four samples prior to the volume movements associated with sampling. The calculated segment concentrations show that the concentration gradient along scala tympani is steeper than the samples suggest. (D) Schematic representation of the volume segments of scala tympani (shown here uncoiled) that comprise the first four samples. The parameters fitted in the simulation were as follows: round window permeability $=14 \times 10^{-8} \mathrm{~m} / \mathrm{s}$; scala tympani clearance half time $=40$ min; volume flow $=1 \mathrm{~nL} / \mathrm{min}$, apically directed; compartment parallel to scala tympani area $=0.3 \mathrm{~mm}^{2}$, accessibility 0.3 re. free diffusion. The volumes in $\mu \mathrm{L}$ for the 10 samples with collection times in seconds shown in parentheses were as follows: 1.06 (18); 1.04 (17); 1.05 (22); 1.04 (21); 1.02 (18); 1.04 (20); 1.06 (24); $1.06(22) ; 1.05$ (19).

to over 40 repeated simulations with parameter adjustments to best-fit simulated samples to the measured concentrations. We therefore developed a less complex but rationally based approach for quantitative interpretation of sequential apical sam-

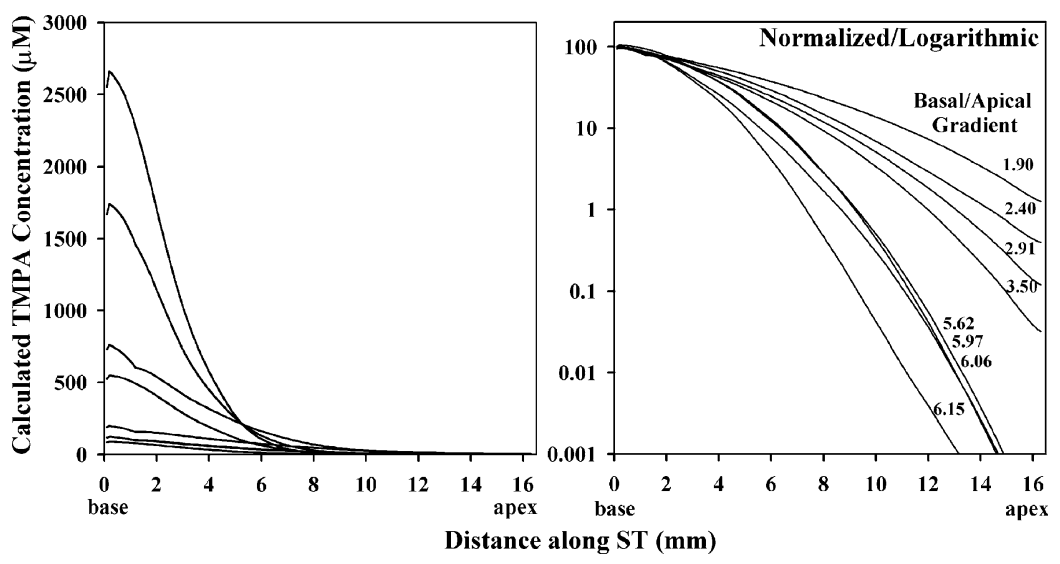

FIG. 5. Concentration profiles along scala tympani (ST) for the eight experiments derived by finite element simulation of each experiment. Left: Concentration profiles shown on a linear scale. Right: The same data, amplitude normalized (setting maximum to 100) and plotted on a logarithmic scale. The basal-apical gradient (in log units) is specified on each curve. 


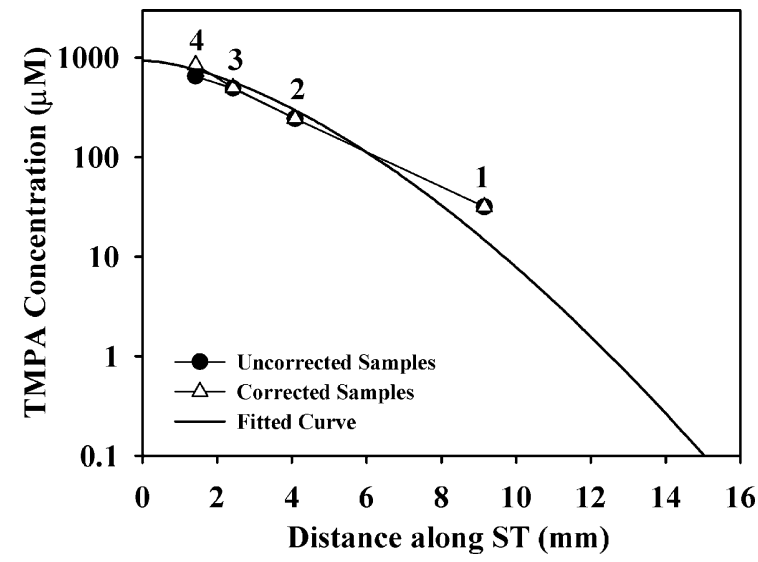

FIG. 6. Analysis of samples using curve fitting. Sample concentrations are plotted based on the location in the cochlea of the sample midpoint. The sample number is shown above each symbol. Basal turn concentrations were corrected for the influence of CSF dilution (open triangles), and a nonlinear function (solid line) was fitted as described in the text. The basal-apical gradient estimated by the fitted curve for this animal was 4.93, which is lower than that estimated by finite-element simulation of the same data (Fig. 4).

the analysis. The sample concentrations plotted as a function of the distance along ST representing their midpoints are shown as solid circles. The necessity to correct samples originating in the basal turn for the interaction with CSF was previously demonstrated (Fig. 3, bottom row). Basal samples were corrected on the basis of their distance from the cochlear aqueduct according to an exponential function derived from simulation of this dilution effect, as described in Methods. For this experiment, the corrected sample concentrations are shown as open

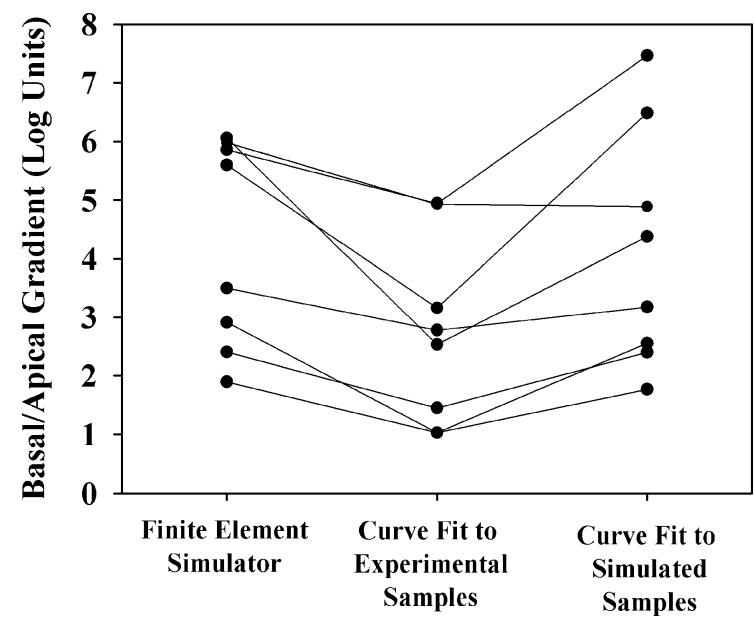

FIG. 7. Summary of the basal-apical gradients calculated by different methods. The gradient calculated by the finite-element simulator was a little greater than that derived by curve fitting, although the difference is small relative to the interanimal variation of gradients. triangles (Fig. 6). Finally, a nonlinear function comparable to the expected profile of TMPA along ST (Fig. 5) was fitted to the four data points. The basal and apical concentrations were estimated from the fitted function to be 937 and $0.011 \mu \mathrm{M}$, respectively, from which the gradient was calculated to be 4.93 (log units).

The gradients established by the curve-fitting method were compared with those obtained by finite element simulations, as shown in Fig. 7. The finite element modeling resulted in somewhat higher estimates of the drug gradient (mean 4.27, SD 1.77) than the curve-fitting method (mean 2.73, SD 1.58) applied to the same sample data. The correlation between the two methods was good, with a correlation coefficient of 0.86 . We initially accounted for the observed differences by the fact that in the curvefitting analysis, there was no correction for the diffusional movements of markers as the more basal samples moved along the cochlea toward the apex during sampling. However, the same curve-fitting method applied to the simulated sample data from the finite element modeling, which included diffusional changes during sampling, did not show similarly low values. Instead, curve-fitting analysis of the simulated samples compared well to the gradients derived from the finite element model (mean 4.14, SD 2.05). The differences between methods did not correlate with the degree to which the first sample value was elevated in some experiments, as shown in Fig. 2 (correlation coefficient 0.09). It did, however, correlate with the rate at which the samples were obtained, with larger differences observed in those animals where the samples were drawn into the pipettes more slowly (correlation coefficient 0.81 ). It is also apparent in Fig. 4A that as the finite-element simulation is fitted to all 10 samples, the fit to the first four samples is not exact. In the example, the first three samples of the simulation show a greater rate of increase than the experimental data, consistent with the finite-element simulation slightly overestimating the TMPA gradient for this region of ST.

\section{DISCUSSION}

The local application of drugs to the inner ear is increasingly being used in clinical treatments of auditory and vestibular disorders (Lustig 2004; Lalwani and McGuire 2005) and in scientific studies of drug effects on the ear (Holley 2005; Rybak and Whitworth 2005). One substantial limitation of local delivery techniques at present is the uncertain or unknown concentration of drugs achieved in the inner ear by specific delivery protocols (Salt and Plontke 2005). Kinetic studies of drug distribution in the inner ear, 
demonstrating the regions in the cochlea the drugs reach and the time courses that they follow in different regions, are essential to conduct phase II clinical trials of new drugs. The target tissues and drug delivery requirements are likely to vary markedly for different clinical applications. For example, when gentamicin is given to Meniere's patients, the goal is to deliver drug to the vestibular system while minimizing the concentration affecting the cochlea. The enhancement of drug gradients along ST would be beneficial in this application by minimizing the drug concentration reaching those regions of the cochlea important for speech frequencies. In contrast, for patients with sudden hearing loss affecting all frequencies of the cochlea, it would be desirable to introduce a drug, such as a steroid, throughout the entire cochlear length. In this scenario, a delivery protocol that minimized drug gradients would be advantageous. The value of the methods presented here is that, for the first time, the magnitude of drug gradients along ST can be measured in a technically straightforward manner while avoiding the technical artifacts that have plagued virtually all ST perilymph measurements in the past.

The existence of longitudinal gradients of drugs along the cochlea have been suggested based on morphological studies (Saijo and Kimura 1984; Imamura and Adams 2003; Stover et al. 1999; Wagner et al. 2005), physiological measures (Chen et al. 2005; Salt and Ma 2001; Salt and Hale 2005), and computer simulations (Plontke et al. 2002; Plontke and Salt 2003). Previously, however, there has not been a methodology capable of documenting or quantifying longitudinal drug gradients. The sequential apical sampling method presented here provides an approach that can be used for any substance or drug for which a very sensitive assay is available. There are significant benefits to the use of repeated, small samples compared with the single, large $(10 \mu \mathrm{L})$ samples taken in our prior study (Salt et al. 2005), although the large sample method may be the only option available with less-sensitive assay methods. The amount of CSF present in large samples (more than $50 \%$ for a $10-\mu \mathrm{L}$ sample) adds some uncertainty to the quantitative interpretation, and no interpretation is possible if the measured drug is also present in CSF. Sequential small samples completely resolve the problem of CSF contamination as the initial samples taken from the apex originate totally from perilymph with negligible CSF contribution. In addition to solving the previously intractable problem of CSF contamination, the analysis of repeated samples further permits the drug gradient along ST to be resolved, rather than having a single value representing the pooled volume of the entire scala. For local drug applications, where drug gradients are likely to exist, this additional information is likely to be of considerable importance.

Sequential sample concentrations displayed graphically provide a qualitative indication of whether a drug gradient exists along the cochlea, as shown in Fig. 3. The concentration values can be further interpreted quantitatively, permitting gradients of drugs with different application systems or with different delivery protocols to be compared. If the drug gradient is of primary interest, then the curvefitting analysis presented here provides a simple approach to determining the spatial origin of the samples and estimating the gradient for the length of the scala. This approach is shown to be more reasonable than the simpler approach of calculating the ratio of the first (apical) and highest (basal) samples, which is likely to underestimate the gradient.

A more detailed analysis of the data is available through finite-element modeling, from which, in addition to drug gradient calculation, RWM permeability properties, drug clearance rate from the scala, and volume flow along the scala can be derived. With the present data set, there were quantitative differences in the longitudinal gradients calculated between the two methods, with the curve-fitting method typically generating a more conservative estimate. The origins of the differences are complex but appear to originate largely from the fact that the finite element model utilized all 10 samples, whereas the curve-fitting method used only the first four samples. It cannot be concluded that the drug gradient determined by one of the methods is necessarily superior to the other.

The round window permeability to TMPA found in the present study $\left(0.93 \times 10^{-8} \mathrm{~m} / \mathrm{s}\right)$ was lower than that found in prior studies (Salt and Ma 2001; Salt et al. 2005). This difference is probably accounted for by the simpler experimental design of the present study. All the prior studies required an ion electrode to be sealed into ST, so that in those studies, the time between opening the auditory bulla and applying drug to the RW membrane was greater. It has been shown that applying dry air to the bulla increases RW membrane permeability (Salt et al. 2005), so it has to be considered possible that opening the middle ear space, and reducing the humidity of gas in contact with the RW membrane, could, over time, affect its permeability properties without any overt manipulation. This could have implications for the local application of drugs to the middle ear in a clinical setting, where variations in surgical protocols between institutions, or in the use of suction in the middle ear, could influence drug levels achieved in perilymph.

The composition of samples taken from the apex in many of the animals showed higher concentrations of TMPA than could be accounted for by passive 
diffusion alone. To account for lower TMPA gradients, another mechanism of TMPA spread was required during the period that the cochlea was loaded with drug, with the apex intact. We have interpreted this additional apical spread of TMPA as resulting from a slow, apically directed movement of perilymph along ST. In a prior study (Salt and Ma 2001), we estimated the longitudinal flow rate along $\mathrm{ST}$ to be $4.4 \mathrm{~nL} / \mathrm{min}$. From the present sample data, the mean flow rate was estimated to be $19 \mathrm{~nL} / \mathrm{min}$. At this rate, it would take over $4 \mathrm{~h}$ to displace the ST contents, a process that would have influence comparable to the TMPA clearance half time of $74 \mathrm{~min}$. For substances that are cleared more slowly from perilymph than TMPA, longitudinal flow at this rate could be an important mechanism of drug dispersal. At present, however, we cannot determine the site of outflow that gives rise to the flow. If physiologic, the efflux must be assumed to occur in scala vestibuli or the vestibule. Artifactual origins cannot yet be ruled out though, as the flow could be caused by evaporation from the bony otic capsule because of the open bulla condition. It is anticipated that chronic drug applications, in which the bulla is reclosed during the application, will ultimately resolve whether this low flow rate is present in the intact animal.

The ability to measure longitudinal gradients of drugs along ST is of great importance in the understanding of drug pharmacokinetics. The distance a drug spreads along the cochlea depends not only on the molecular size, but also on how rapidly the substance is cleared from the perilymph to other compartments (such as blood, other scalae, etc.). If the substance is cleared rapidly, then a steady state is rapidly established in which drug diffusion along the scala is balanced by clearance to the extent that the drug may never reach the apical regions in appreciable concentration. Measurement of the drug gradient once a steady state has been reached after prolonged application allows the clearance rate to be quantified. As clearance is the most important parameter affecting drug distribution, this would be a major step forward in our quantitative understanding and our ability to simulate drug kinetics in the ear.

The analysis of large samples from the apex (Salt et al. 2005) and both of the analytic methods used here make the assumption that there is no drug present in the CSF. The simulations of the drug decline in the later samples of each experiment validate this assumption for the present application protocol. However, it is accepted that there may be specific delivery protocols (such as combined local and systemic delivery) where a zero concentration of drug in CSF cannot be assumed. In such cases, the finite element simulation could be modified to allow the CSF concentration of drug to be varied to fit the observed rate of marker decline in later samples, thus allowing data of this type to be interpreted.

In conclusion, sequential sampling of perilymph from the cochlear apex allows drug levels along ST to be quantified without the artifacts because of CSF contamination of samples taken from the basal turn that have been shown to have distorted many pharmacokinetic studies in the past (Salt et al. 2003).

\section{ACKNOWLEDGMENTS}

The technical contributions of Jared Hartsock in the simulation and analysis of data in this study are appreciated. The study was supported by research grant RO1 DC01368 from the National Institute on Deafness and Other Communication Disorders, National Institutes of Health.

\section{REFERENCES}

Chen Z, Kujawa SG, McKenna MJ, Fiering JO, Mescher MJ, Borenstein JT, Leary Swan EE, Sewell WF (2005) Inner ear drug delivery via a reciprocating perfusion system in the guinea pig. J. Control. Release 110:1-19.

Ghiz AF, Salt AN, DeMott JE, Henson MM, Henson OW Jr, Gewalt SL (2001) Quantitative anatomy of the round window and cochlear aqueduct in guinea pigs. Hear Res. 162:105-112.

Hara A, Salt AN, Thalmann R (1989) Perilymph composition in scala tympani of the cochlea: influence of cerebrospinal fluid. Hear Res. 42:265-271.

Hoffer ME, Balough B, Henderson J, DeCicco M, Wester D, O'LeARY MJ, Kopke R (1997) Use of sustained release vehicles in the treatment of Meniere's disease. Otolaryngol. Clin. North Am. 30:1159-1166.

Holley MC (2005) The auditory system, hearing loss and potential targets for drug development. Drug Discov. Today 10:12691282.

Imamura S, Adams JC (2003) Distribution of gentamicin in the guinea pig inner ear after local or systemic application. J. Assoc. Res. Otolaryngol. 4:176-195.

Lalwani AK, McGuire JF (2005) Pharmacologic treatment of the cochlea and labyrinth. In: Cummings CE et al. (eds) Otolaryngology-Head and Neck Surgery, 4th ed., Philadelphia, Elsevier Mosby.

Lustig RL (2004) The history of intratympanic drug therapy in otology. Otolaryngol. Clin. North Am. 37:1001-1018.

Moscovitch DH, Gannon RP, Laszlo CA (1973) Perilymph displacement by cerebrospinal fluid in the cochlea. Ann. Otol. Rhinol. Laryngol. 82:53-61.

Ohyama K, Salt AN, Thalmann R (1988) Volume flow rate of perilymph in the guinea-pig cochlea. Hear Res. 35:119-130.

Plontke SKR, SALt AN (2003) Quantitative interpretation of corticosteroid pharmacokinetics in inner ear fluids using computer simulations. Hear Res. 182:34-42.

Plontke SK, Wood AW, SAlt AN (2002) Analysis of gentamicin kinetics in fluids of the inner ear with round window administration. Otol. Neurotol. 23:967-974.

Rybak LP, Whitworth CA (2005) Ototoxicity: therapeutic opportunities. Drug Discov. Today 10:1313-1321.

SAIJO S, KIMURA RS (1984) Distribution of HRP in the inner ear after injection into the middle ear cavity. Acta Otolaryngol. 97:592610 . 
Salt A, Hale S (2005) Longitudinal distribution of drugs in perilymph assessed by cochlear action potentials. 28th midwinter research meeting of the ARO, New Orleans (Abstract).

SALt AN, MA Y (2001) Quantification of solute entry into cochlear perilymph through the round window membrane. Hear Res. 154:88-97.

Salt AN, Plontke SK (2005) Local inner ear drug delivery and pharmacokinetics. Drug Discov. Today 10:1299-1306.

SALt AN, STOpp PE (1979) The effect of cerebrospinal fluid pressure on perilymphatic flow in the opened cochlea. Acta Otolaryngol. 88:198-202.

SAlt AN, Vora AR (1991) Calibration of ion selective microelectrodes for use with high levels of interfering ions. J. Neurosci. Methods 38:233-237.

Salt AN, Inamura N, Thalmann R, Vora AR (1991) Evaluation of procedures to reduce fluid flow in the fistulized guinea pig cochlea. Acta Otolaryngol. 111:899-907.
Salt AN, Kellner C, Hale S (2003) Contamination of perilymph sampled from the basal cochlear turn with cerebrospinal fluid. Hear Res. 182:24-33.

Salt AN, Hale SA, Plontke SKR (2005) Perilymph sampling from the cochlear apex: a reliable method to obtain higher purity perilymph samples from scala tympani. J. Neurosci. Methods (doi: 10.1016/j.jneumeth.2005.10.008).

Scheibe F, Haupt H, Bergmann K (1984) On sources of error in the biochemical study of perilymph (guinea pig). Arch. Otorhinolaryngol. 240:43-48.

Stover T, Yagi M, Raphael Y (1999) Cochlear gene transfer: round window versus cochleostomy inoculation. Hear Res. 136:124130.

Wagner N, Caye-Thomasen P, Laurell G, Bagger-Sjoback D, Thomsen J (2005) Cochlear hair cell loss in single-dose versus continuous round window administration of gentamicin. Acta Otolaryngol. 125:340-345. 